\title{
Heat transfer in heated industrial premises with using radiant heating system
}

\author{
Tatiana A. Nagornova ${ }^{1, *}$, Dmitriy A. Lipchinskiy ${ }^{1}$ \\ ${ }^{1}$ National Research Tomsk Polytechnic University, 634050 Tomsk, Russia
}

\begin{abstract}
The results of mathematical modeling of heat transfer processes in a closed air volume surrounded by enclosing constructions, heated by supplying energy to the upper contour of gas infrared radiators are represented. Regimes of turbulent natural conjugate convection in the region bounded by solid walls are investigated. Two-dimensional nonstationary problem is solved in the framework of the Navier -Stokes equations for gas and thermal conductivity for solid walls. Nonstationary processes of heat propagation in course of time and essential heterogeneity of temperature fields and heat fluxes are established.
\end{abstract}

\section{Introduction}

In modern industrial production in many cases only a small part of the area used for workshops equipment maintained by the staff. Accordingly, the use of traditional systems of convective heating for the heating of industrial premises is not in most cases economically justified. Expedient use of infrared gas radiators for optimal thermal regime in certain areas large space for production purposes.

Introduction in practice of heat radiation heating systems until recently, severely limited by including in connection with the lack of science-based technologies for supplying heat energy from radioactive sources to heat objects. Thus, in particular, are not carried out any theoretical or experimental investigation of the laws of heat transfer in an area surrounded by special walling. No information about the temperature fields bounded by the outer contour of the air volume with the radiation flux at the upper boundary of the heating region.

The basis of the known methods for calculating thermal regimes objects heated by infrared gas emitters are balanced mathematical models [1]. Thus, as a rule, is not considered as heterogeneity and non-stationary temperature fields of the object. At the same time, these two factors in most real options radiant heating systems can play a decisive role. Informed choice of technology management heating industrial premises is impossible without analysis of temperature fields of the heating region. The purpose of this research is mathematical modeling of heat transfer in a closed air volume heated by supplying energy to the upper contour of gas infrared radiators.

* Corresponding author: tania@tpu.ru 


\section{Physical and mathematical statement of the problem}

The region of a rectangular cross-section is considered. At the upper boundary region is located the infrared source. The intensity of the radiation is uniformly distributed along the transverse coordinate $X$ (Fig. 1).

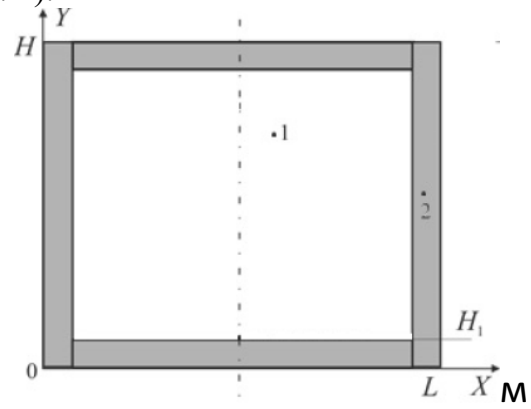

Fig. 1. Area of solution of the problem under consideration: 1 - air; 2 - enclosing structures.

The space inside the considered area is filled with air (1), which is surrounded by heatconducting walls of finite thickness (2). Source of radiant heating in the top of the decision does not stand - it was assumed that the thickness is small compared with the characteristic dimensions of the field. Problem was considered in the plane formulation for a region with a heating zone, located at the upper boundary. The air was assumed optically transparent medium. The main mechanisms of heat transfer were considered: in the gas turbulent natural convection and thermal conductivity, in the enclosing structures - thermal conductivity. When the problem was assumed that the energy of infrared emitter proceeds to boundary $H_{1}$, which divides lower concrete base (2) and the heating region (1). Heat from the gas in the vertical enclosing structure (2) is performed due to turbulent convection and conduction.

As a basic system of differential equations, is accepted mathematical model [2-4] has been developed to describe the processes of convection and heat conduction in a closed rectangular region with heat-conducting walls and a local heating source at the lower boundary and developed later for solving heat transfer problems under radiant heating [5]. The process of heat transfer in the analyzed solution region (Fig. 1) is described by a system of non-stationary two-dimensional partial differential equations [5].

Numerical analysis is performed to change the numbers in the range from $10^{6}$ to $\mathrm{Gr}$ $10^{11}$. To solve the problem algorithm was used [3-5], developed for the numerical solution of natural convection in a closed rectangular regions. Turbulence in the flow of heated air taken into account in accordance with the basic provisions of the [6-8] change viscosity with increasing air velocity. Calculation of viscosity is carried out within the Prandtl model [6-8].

\section{Analysis of numerical simulation results}

Numerical studies were carried out at the values of the heat flux arriving at the $H_{1}$ surface (Table 1).

Fig. 2-3 shows the results of numerical simulations illustrating the basic laws of heat transfer in the system heating using gas infrared heaters.

Isotherms Fig. $2 a, c, e$ illustrate the process of cooling the heated air near the floor of the concrete mass at relatively cold reduction along the vertical walls. This results in a symmetrical circulation of heated air for $X=0.5$ (Fig. $2 b, d, f$ ). 
Table. 1. The values of the heat flux over the surface of the concrete base in the section $Y=H_{1}$ for $L / 2<X<0.875 L$ (Fig. 1).

\begin{tabular}{|l|l|l|l|l|l|l|l|}
\hline Сечение & $\mathrm{X}=0.5 \mathrm{~L}$ & $\mathrm{X}=0.563 \mathrm{~L}$ & $\mathrm{X}=0.685 \mathrm{~L}$ & $\mathrm{X}=0.688 \mathrm{~L}$ & $\mathrm{X}=0.75 \mathrm{~L}$ & $\begin{array}{l}\mathrm{X}=0.813 \\
\mathrm{~L}\end{array}$ & $\begin{array}{l}\mathrm{X}=0.875 \\
\mathrm{~L}\end{array}$ \\
\hline $\mathrm{q}, \mathrm{W} / \mathrm{m}^{2}$ & 190.5 & 180.2 & 182.1 & 172.2 & 134.2 & 122.2 & 102.1 \\
\hline
\end{tabular}
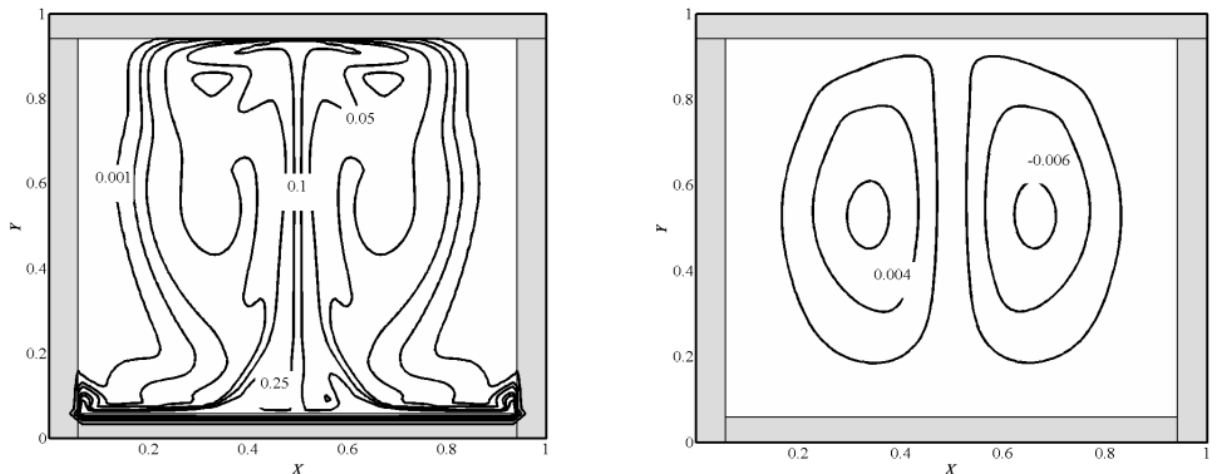

$a$

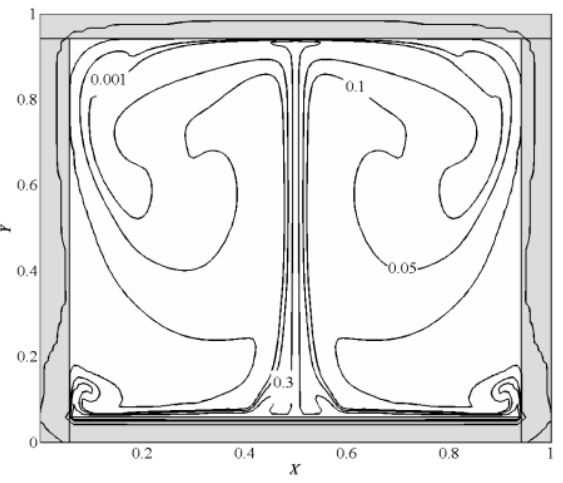

$b$
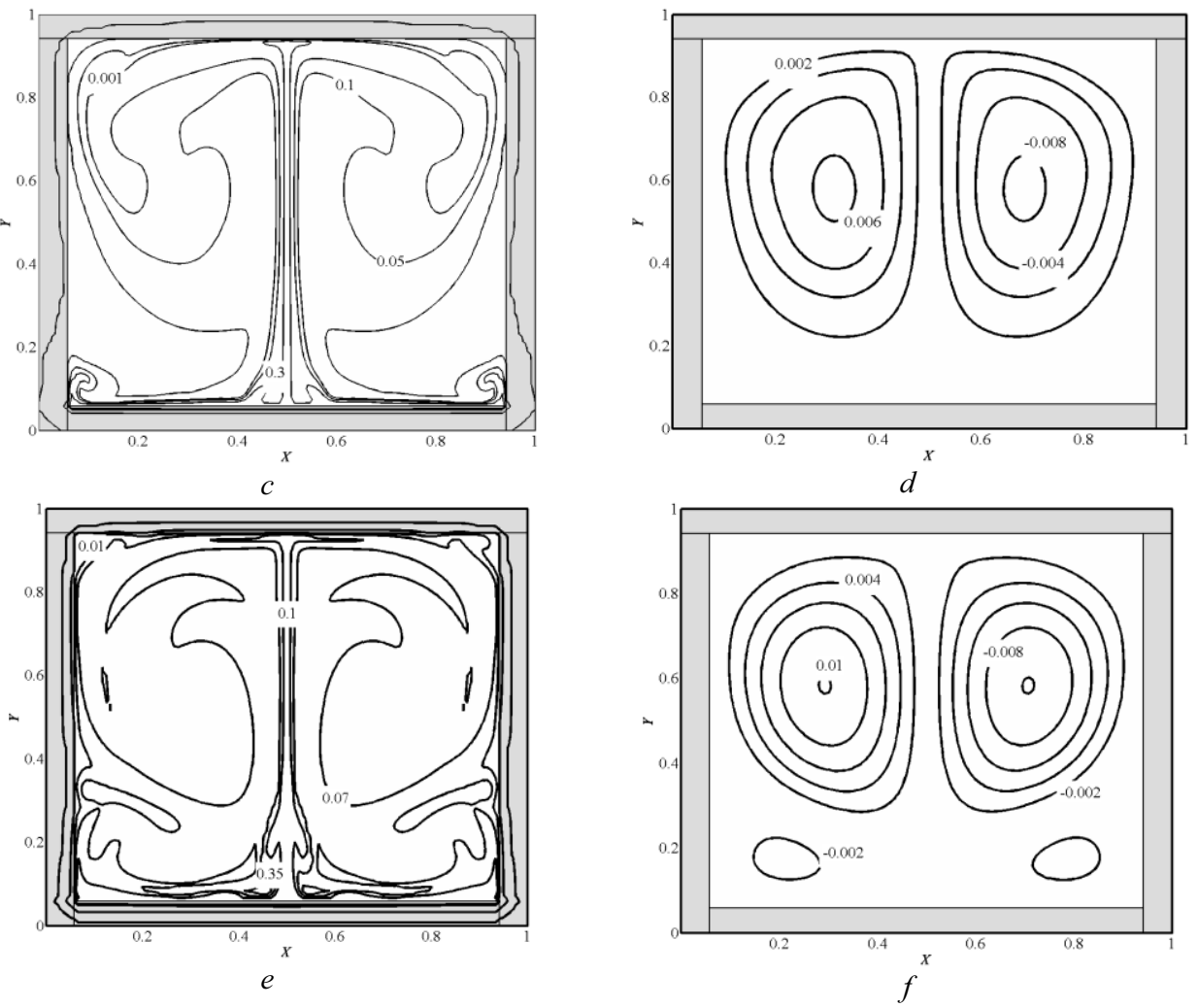

Fig. 2. Fields of temperatures $(a, c, e)$ and the contours of the stream function $(b, d, f)$ for the model of natural convection in a closed area with radiative heating of the lower boundary "gas - solid wall" for different values of $\tau: \tau=70(a, b), \tau=120(c, d), \square \tau=150(e, f)$

Figures 2 and 3 well illustrate the nonstationary character of the heat transfer process in the considered rather typical solution region. Well as a comparison of the provisions specific isotherms in Fig. $2 a, c, e$ and temperature distributions (Fig. 3) allows you to make 
a conclusion about the high values of the temperature gradients, both in height and in the transverse direction. Based on the above we can conclude essentially stationary in time and inhomogeneous fields of temperature and heat flow in the heating gas infrared emitters.

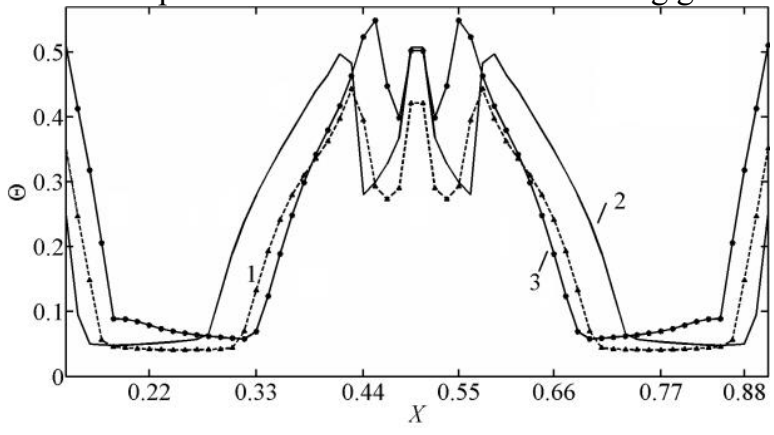

$a$

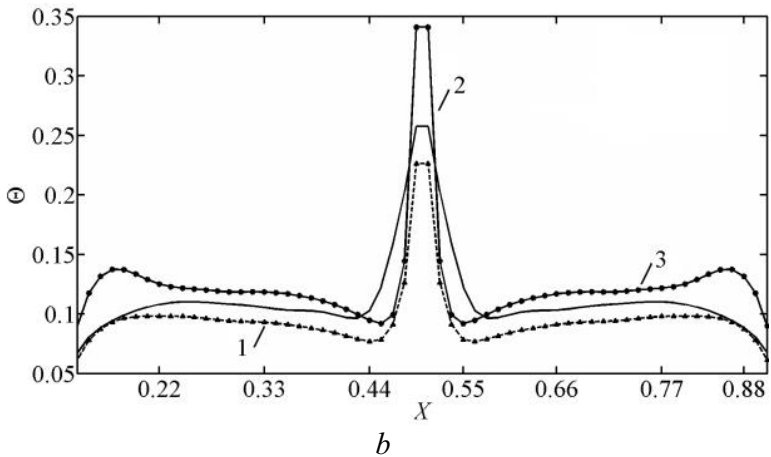

Fig. 3. Temperature profiles in the cross section $Y=0.1(a)$ and $Y=0.5(b)$ at different time points: 1 $-\tau=70 ; 2-\tau=120 ; 3-\tau=150$.

The regularities are due to the specifics of heat transfer processes in the production premises, the height of which is usually $4-5 \mathrm{~m}$ or more. With such large volumes of heated air and cooling surface area (building envelope), coming from the surface of a heated concrete floor intensively cooled air as it rises to the top of the heated space. Heatsink on vertical surfaces intensifies circulation flow, increasing the rate of removal of accumulated in a layer of concrete floor heat, on the one hand. But, from other side in this case the substantial part of the energy is expended on heating of the vertical enclosing constructions.

It may be noted that the distributions (Fig. 3) generally correspond fairly well established experimentally [11] in a similar temperature dependence on the transverse coordinate in the typical cross-section.

The results obtained are objective grounds for withdrawal of the need to meet the challenges of turbulent natural convection and heat conduction within at least flat models in the evaluation of the effectiveness of local radiation heating the working areas of industrial premises.

The solution of problems of free convection is advantageously carried out in the combined formulation based on the heat sink in the building envelope and accumulation in them of energy. Balanced type model [1] thus can serve as a tool for the lower estimates of the energy required for the effective use of radiant heating local jobs.

It may be noted that in spite of the low thermal conductivity of most traditional building materials used for the production of walling (brick, concrete, reinforced concrete), the characteristic times of the processes involved are so large (up to 10 hours), high heat 
capacity materials that the walls and floor leads to the accumulation of too much fraction of the energy dissipated in the heating. These losses are likely, can be significantly reduced by using heat-insulating coatings on vertical surfaces walling industrial premises.

\section{Conclusion}

According to the results of solving the above problem of heat transfer in the local object of heat supply, heated using gas infrared radiators can conclude substantial heterogeneity and non-stationary temperature fields in the region heated by turbulent convection and conduction. The theoretical investigation also constitute grounds for withdrawal of the feasibility solutions conjugate heat transfer problems in the analysis of thermal conditions of local heating objects using radiant heating systems.

The work was supported by the Russian President's grant (Scientific School project 7538.2016.8).

\section{References}

1. G. Gong, J. Liu, X. Mei, Energy Buildings, 138, 193 (2017)

2. G.V. Kuznetsov, M.A. Sheremet, Fluid Dynamics, 41 (6), 881 (2006)

3. G.V. Kuznetsov, M.A. Sheremet, Comput. Therm. Sci. 1 (3), 341 (2009)

4. V.I. Maksimov, T.A. Nagornova, I.A. Shestakov, EPJ Web Conf. 82, 01048 (2015)

5. G.V. Kuznetsov, N.I. Kurilenko, V.I. Maksimov, G.Ya. Mamontov, T.A. Nagornova, J. Eng. Phys. Thermophys. 86 (3), 519 (2013)

6. D.S-K. Ting, Basics of engineering turbulence (Academic Press, 2016)

7. T. Cebeci, Analysis of Turbulent Flows with Computer Programs (Elsevier Ltd., Butterworth-Heinemann, 2013)

8. J. Blazek, Computational fluid dynamics. Principles and Applications (Elsevier Ltd., Butterworth-Heinemann, 2015) 